\title{
A Fluorescence Assay for Evaluating the Permeability of a Cardiac Microvascular Endothelial Barrier in a Rat Model of Ischemia/reperfusion
}

\author{
Ruijie Tang ${ }^{*}, 1$, Kaihao Wang ${ }^{*}, 1$ Yuyan Xiong ${ }^{1}$, Jian Meng ${ }^{2}$, Yuejin Yang ${ }^{1}$ \\ ${ }^{1}$ State Key Laboratory of Cardiovascular Disease, Department of Cardiology, Fuwai Hospital, National Center for Cardiovascular Diseases, Chinese \\ Academy of Medical Sciences and Peking Union Medical College ${ }^{2}$ State Key Laboratory of Cardiovascular Disease, Fuwai Hospital, National Center for \\ Cardiovascular Diseases, Chinese Academy of Medical Sciences and Peking Union Medical College \\ *These authors contributed equally
}

\section{Corresponding Author}

Yuejin Yang

yangyuejin_fw@126.com

\section{Citation}

Tang, R., Wang, K., Xiong, Y., Meng, J., Yang, Y. A Fluorescence Assay for Evaluating the Permeability of a Cardiac Microvascular Endothelial Barrier in a Rat Model of Ischemia/ reperfusion. J. Vis. Exp. (172), e62746, doi:10.3791/62746 (2021).

\section{Date Published}

June 30, 2021

\section{DOI}

$10.3791 / 62746$

URL

jove.com/video/62746

\section{Abstract}

Revascularization therapies for culprit arteries, regardless of percutaneous coronary intervention and coronary artery bypass grafting, are considered the best strategy for improving the clinical prognosis of patients with acute coronary syndrome (ACS). Nonetheless, myocardial reperfusion following effective revascularization can trigger significant cardiomyocyte death and coronary endothelial collapse, known as myocardial ischemia/reperfusion injury (MIRI). Usually, endothelial cells and their intercellular tight junctions cooperatively maintain the microvascular endothelial barrier and its relatively low permeability but fail in reperfusion areas.

Microvascular endothelial hyperpermeability induced by ischemia/reperfusion (IR) contributes to myocardial edema, increased infiltration of pro-inflammatory cells, and aggravated intramyocardial hemorrhage, which may worsen the prognosis of ACS. The tracer used in this study-70,000 Da FITC-dextran, a branched glucose molecule labeled by fluorescein isothiocyanate (FITC)-appears too large to infiltrate the cardiac microvascular endothelium in normal conditions. However, it is capable of infiltrating a broken barrier after MIRI. Thus, the higher the endothelial permeability is, the more FITC-dextran accumulates in the extravascular intercellular space. Thus, the intensity of fluorescence from FITC can indicate the permeability of the microvascular endothelial barrier. This protocol takes advantage of FITC-dextran to evaluate the cardiac microvascular endothelial barrier functionally, which is detected by an automated quantitative pathology imaging system. 
The fact that an increasing number of patients with coronary heart diseases have benefited from revascularization therapies for decades does not negate the fatal consequences of MIRI. As described by Yellon and Hausenloy, approximately $30 \%$ of the final infarct size can be attributed to $\mathrm{MIRI}^{1}$. Cardiac microvascular endothelial dysfunction is the core mechanism underlying MIRI and the "no-reflow" phenomenon and is the target of many cardioprotective agents and classical cardioprotective pathways $^{2}$. Moreover, endothelial hyperpermeability of microvessels can trigger myocardial edema ${ }^{3}$ and the overinfiltration of pro-inflammatory cells, including neutrophils ${ }^{4}$, macrophages, and erthrocytes ${ }^{5}$.

MIRI-induced oxidative stress and overexpressed proinflammatory cytokines, such as the members of the tumor necrosis factor family ${ }^{6}$ and the interleukin family ${ }^{7}$, activate signaling pathways of cell apoptosis to accelerate the death of ischemic myocardium. Erythrocytes penetrate the vulnerable endothelial barrier and accumulate in the extracellular matrix (ECM) to form intramyocardial hematoma ${ }^{8}$. Macrophage- and neutrophil-derived matrix metalloproteinases disassemble collagen in the ECM, which increases the risk of mechanical complication and post-infarction ventricular aneurysm ${ }^{9}$. Thus, the permeability of the endothelial barrier is a crucial sign for the prognostic evaluation of acute myocardial infarction and marks the severity of MIRI to some degree.

FITC-dextran is an array of polysaccharides comprised of different lengths of branched glucose molecules of molecular weight ranging from $4,000 \mathrm{Da}$ to $70,000 \mathrm{Da}$. It is widely used as the standard tracer to demonstrate the permeability of cell membranes, functional physiological barriers such as the blood-brain barrier ${ }^{10}$, and mucosal membranes ${ }^{11}$ under specific treatments and pathological conditions. The permeability of one specific membrane varies for different substances according to their molecular weights. Usually, albumin is restricted to cardiac microvessels; however, it can penetrate the endothelium in the early stage of IR, causing myocardial edema. As the molecular weight of albumin is approximately $68,000 \mathrm{Da}, 70,000 \mathrm{Da}$ FITC-dextran would be a suitable agent to demonstrate endothelial hyperpermeability after IR. Moreover, dextran has been widely adopted to maintain the blood volume and colloid osmotic pressure of shock patients, and FITC-labeled tracers are used in numerous in vivo or in vitro studies with few adverse effects.

In addition to FITC-dextran, endothelial permeability has been measured with Evans Blue (EB) staining. After injection into the blood circulation, EB binds to serum albumin tightly and forms a complex ${ }^{12}$. Thus, this EBalbumin complex, not EB alone, can be used for the measurement of permeability, which means that the accuracy of such an EB-based method can be influenced by different concentrations of serum albumin in experimental subjects. Additionally, EB has been reported to poison the organs of experimental animals, especially the heart, liver, and lungs, via cellular degeneration and increased vascular permeability ${ }^{13}$. Endothelium undergoing ischemia/ reperfusion would be more sensitive to such detrimental effects than normal endothelium, which can confound the significance of experimental results.

Compared to EB dye, FITC-dextran is exogenous, and the binding affinity to albumin is negligible. Hence, FITCdextran remains in the free state and is not bound by macromolecules, such as albumin and tissue proteins. Thus, if administered according to body weight, FITC-dextran can work at equal blood concentration and is not affected by individual differences in serum albumin concentration 
and blood volume. Tongxinluo ( $\mathrm{TXL}$ ), a traditional Chinese medicine, was used to test the diagnostic sensitivity of this method. TXL reduces MIRI by maintaining the endothelial cellular ultrastructure and endothelial barrier integrity ${ }^{14}$. TXL is also used to cure angina pectoris and improve myocardial no-reflow after infarction clinically ${ }^{15}$. It contains Radix ginseng, Buthus martensi, Hirudo, Eupolyphaga seu steleophaga, Scolopendra subspinipes, Periostracum cicadae, Radix paeoniae rubra, Semen ziziphi spinosae, Lignum dalbergiae odoriferae, Lignum santali albi, and Borneolum syntheticum. TXL has been shown to protect the microvascular endothelium via the MEK/ERK pathway ${ }^{16}$ and endothelial nitric oxide synthase-mediated mechanisms ${ }^{5,17}$ and alleviate ischemia/reperfusion injury (IRI) of organs ${ }^{18}$, especially the heart, in a dose-dependent manner. Here, rats were fed with $0.5 \mathrm{~g} / \mathrm{kg} \mathrm{TXL}$ powder (an equivalent dose to that used clinically in humans) as described ${ }^{19}$. This paper provides a method to functionally assess the endothelial barrier of cardiac microvessels after IR via the measurement of the total fluorescence intensity of extravasated 70,000 Da FITC-dextran compared to EB.

\section{Protocol}

Animal experiments were performed in accordance with the "Guide for the Care and Use of Laboratory Animals" issued by the US National Institutes of Health (Bethesda, MD, USA, NIH Publication No. 85-23, revised 1996) and the "Regulation to the Care and Use of Experimental Animals" of the Beijing Council on Animal Care (1996). The study protocol was approved by the Care of Experimental Animals Committee of Fuwai Hospital. Male SPF-class Sprague-Dawley rats (SD rats) weighing 150-180 g were used in this study.

\section{Preparation of reagents, materials, and equipment}

1. Prepare reagents and solutions including $70 \%$ ethanol, FITC-dextran $(70,000 \mathrm{Da}$, dissolved in ice-cold saline to make $10 \% \mathrm{w} / \mathrm{v}$ solution for use), hair removal cream, mounting medium with 4',6-diamidino-2-phenylindole (DAPI, $0.5-10 \mu \mathrm{g} / \mathrm{mL}$ ), $1 \% \mathrm{w} / \mathrm{v}$ EB/saline solution, and $5 \%$ $\mathrm{w} / \mathrm{v}$ pentobarbital sodium/saline solution (stored in $4{ }^{\circ} \mathrm{C}$ in the dark).

2. Sterilize all surgical tools and materials in advance, including surgical tape; gauze pads; surgical gloves; 16 G endotracheal tube; insulin syringe; operating scissors; large, curved dissecting forceps; straight dissecting forceps; straight mosquito forceps; curved mosquito forceps; retractors (30 mm Spread); $5 \mathrm{~cm}$ microneedle holders; 3-0 polypropylene suture (taper needle); and 6-0 polypropylene suture (round needle).

3. Check and ensure that all equipment, including the constant temperature warming system, illumination system, ventilator, automated quantitative pathology imaging system, and ultraviolet freezing slicer, are in good condition.

\section{Preparing the rats for the surgical procedures}

1. Divide all SD rats into Sham group, IR group, and TXL group (IR+TXL group) randomly.

NOTE: In this protocol, six rats were randomly assigned to each group, $n=6$.

2. Weigh each SD rat, record the weight, and calculate the dose of TXL to be administered for each rat. 
3. Administer ibuprofen at a dose of $30 \mathrm{mg} / \mathrm{kg}$ by gavage to each rat at least $12 \mathrm{~h}$ prior to thoracotomy to alleviate preoperative pain and distress.

4. Administer a superfine powder of TXL at a dose of $0.5 \mathrm{~g} /$ $\mathrm{kg}$ by gavage $12 \mathrm{~h}$ and $1 \mathrm{~h}$ to each SD rat in the IR+TXL group before anesthetization, as described ${ }^{19}$.

5. Disinfect the operating table with $70 \%$ ethanol and place the sterilized surgical tools and materials on the disinfected surface.

6. Adjust the temperature of the constant temperature warming system to maintain the body temperature of the rats at $37 \pm 1{ }^{\circ} \mathrm{C}$.

\section{Establish a rat model of cardiac ischemia/ reperfusion}

1. Inject $5 \% \mathrm{w} / \mathrm{v}$ pentobarbital sodium/phosphate-buffered saline (PBS) solution at a dose of $50 \mathrm{mg} / \mathrm{kg}(0.1 \mathrm{~mL} / 100$ g body weight) into the abdominal cavity to anesthetize the rat. Prepare the surgical site by carefully removing the fur from the neck to the chest by shaving or using a depilatory cream.

2. Gently adjust the position of the tongue as needed with sterilized tweezers and wipe respiratory secretion with sterilized cotton balls. Keep the glottis clearly in view and insert an endotracheal tube into the trachea gently.

3. Connect the endotracheal tube to a ventilator circling at $65-80$ breaths per minute and adjust the tidal volume at 4-5 $\mathrm{mL}$ by fixing the rat's head and limbs.

4. Place a sterile drape over the rat, disinfect the operating area with tincture of iodine followed by $70 \%$ ethanol, and repeat this procedure three times.
5. Make a left parasternal incision at the level of the fourth intercostal space to expose the left musculus pectoralis major and pectoralis minor.

6. Dissect the fascia between the musculus pectoralis major and pectoralis minor using a blunt dissection technique to expose the left fourth intercostal space.

NOTE: Avoid excessive tissue damage and vascular injury that will cause severe bleeding and postoperative death.

7. Dissect the intercostal muscles, position a chest retractor within the fourth intercostal space, and then spread the ribs.

8. Tear the pericardium and remove the attached adipose tissue.

NOTE: Avoid damaging other organs with sharp surgical instruments, especially the lungs. Damage to the lungs can result in the development of pneumothorax and hemopneumothorax during the operation, causing cacoethic consequences ranging from hemodynamic disorders to reduced animal survival.

9. Manipulate the position of the thymus and expose the ascending aorta and left atria gently. Identify and ligate the proximal left anterior descending (LAD) artery transiently for 45 min with a 6-0 polypropylene suture (see Figure 1 A-D).

NOTE: This procedure is identical for sham control rats except that the LAD is not ligated.

10. Remove the chest retractor and draw the ribs together in an interrupted suture pattern with a 3-0 polypropylene suture.

11. Restore negative intrathoracic pressure to ensure effective breathing. 
NOTE: Two methods can be used to restore negative intrathoracic pressure: i) before closing the chest cavity, researchers can plug up the air outlet of the ventilator for 2 cycles to inflate the lungs. ii) The air remaining in the chest cavity can be drawn out with a syringe after the intercostal space has been closed.

12. After hair removal and disinfection, make an incision in the right lower limb and dissect the superficial fascia from the right femoral vein.

13. Loosen the ligation and check the respiration and circulation conditions after inducing myocardial ischemia for $45 \mathrm{~min}$.

14. Inject $10 \%(w / v)$ FITC-dextran/saline solution into the femoral vein at $0.15 \mathrm{~mL} / 100 \mathrm{~g}$ body weight, and press the femoral vein for 1 min with sterile cotton balls to stanch the bleeding (Figure 2A,B).

NOTE: Alternatively, inject $1 \% \mathrm{w} / \mathrm{v} \mathrm{EB} /$ saline solution into the femoral vein at a dose of $0.5 \mathrm{~mL} / 100 \mathrm{~g}$ body weight.

15. Sew up the incisions with a 3-0 polypropylene suture and disinfect the incisions, followed by postoperative analgesia with ibuprofen, as described above.

\section{Preparation of frozen tissue slices for fluorescence measurement}

1. Inject $5 \% \mathrm{w} / \mathrm{v}$ pentobarbital sodium/PBS solution at a dose of $150 \mathrm{mg} / \mathrm{kg}$ (0.3 mL/100 g body weight) intraperitoneally to anesthetize the rats deeply after $3 \mathrm{~h}$ of myocardial reperfusion.

2. Slit the abdominal cavity and expose the aorta abdominalis and vena cava (see in Figure 3A).

3. Inject $50 \mathrm{~mL}$ of ice-cold PBS into the vena cava steadily, cut the aorta abdominalis to flush blood from the myocardium, and harvest the heart.
NOTE: Observe changes in the lungs and liver. A sweating liver suggests that saline is accurately injected into the vena cava (see in Figure $\mathbf{3 C}$ ), and a pale lung is considered a sign of a well-perfused myocardium (see in Figure 3B).

4. In a dark room, rinse the heart in ice-cold saline or PBS gently to remove any remaining blood and FITC-dextran (or EB) from the chambers of the heart.

5. Cut off the heart from the ligation and discard the myocardium above the ligation.

6. Embed the heart in molds with embedding compound followed by storage at $-80^{\circ} \mathrm{C}$.

7. Unload the frozen tissues from the embedding molds and load them on sample holders in a prechilled $-20^{\circ} \mathrm{C}$ chamber of a freezing slicer.

8. Adjust the thickness of a single slice to $5-8 \mu \mathrm{m}$ and slice the tissue at approximately the midpoint of the base-apex axis (e.g., the middle of the ischemia).

9. Load the sliced tissue on adhesive microscope slides and seal each slice with one drop (approximately $40 \mu \mathrm{L}$ ) of fluorescent mounting medium containing DAPI.

10. Keep the slides in a dry and dark box.

NOTE: If the slides cannot be scanned immediately, store them in a dry, cool, and dark place to prevent the formation of condensate water and fluorescence quenching.

\section{Fluorescence measurement and data analysis}

1. Set up the imaging system and check the software connected to the scanner.

2. Load the slides in the slide shelves and insert them into the scanner. 
3. Open the slide scanner software (see the Table of Materials) and set up a new exposure protocol by clicking on Edit Protocol | New....

NOTE: If the scanner or its affiliated software is not available, a laser confocal microscope (see the Table of Materials) or other ordinary slide scanners with the fluorescence mode (see the Table of Materials) may be an alternative to the automated quantitative fluorescence scanner.

4. Rename the protocol and study, and select the fluorescence mode.

5. Define Whole Slide Scan | Pixel Resolution and Multispectral Regions | Pixel Resolution as $0.25 \mu \mathrm{m}$ (40x) respectively.

6. Click on Edit Filters and Bands... to select the Whole Slide Scan Filters column and the Multispectral Region Bands column.

NOTE: If EB is used, select Whole Slide Scan Filters I DAPI and Texas Red or AF495. If FITC-dextran is used, select Multispectral Region Bands | DAPI and FITC or AF488.

7. Click on Edit Exposures... and load the carrier.

8. Select the target slot and click on Take overview.

NOTE: This takes a brightfield scan of each heart section. Select the myocardium for focusing or select the area blindly.

9. Select the target slide, adjust Stage Height to approximately $-50 \mu \mathrm{m}$, and click on Auto Focus.

10. Click on Whole Scan Exposure(ms)-DAPI Autoexpose.

NOTE: The exposure time of DAPI for each slide does not have to be the same because images with DAPI are used to illustrate cross-sections of the heart, which are convenient for outlining the area of the myocardium but not to calculate fluorescence density.

11. Click on Whole Scan Exposure(ms)-FITC | Autoexpose.

NOTE: Click on Whole Scan Exposure(ms)-Texas Red I Autoexpose if using EB. It is important to remember and ensure that the exposure time of FITC or Texas Red is the same in all slide scans.

12. Click on Auto Focus again and ensure that the slide is in focus.

13. Click on Back and save the protocol.

14. Repeat steps 5.7-5.14 for each slide.

15. Return to the home page and click on Scan Slides.

16. Select a slot, rename all the slides, and select the corresponding exposure protocol for each slide.

17. Scan the slides and capture the images.

18. Open the images with slide viewer software for the scans (see the Table of Materials).

19. Magnify all the images to a relatively equal extent.

20. Snapshot and save images as .tiff.

NOTE: It is better to save the images with and without scale bars at the same time. Images with scale bars can be used for publication, whereas those without scale bars can be used for statistical analysis.

21. Open ImageJ software, click on Analyze, and select Set Measurement.

22. Select Area | Integrated density.

23. Open the image in the FITC channel with Image J.

24. Click on Analyze again and record the numerical value of integrated density (IntDen) that appears. 
NOTE: As scale bars in the images can be mistaken for grayscale of fluorescence by computers, use images without scale bars to measure IntDen.

25. Close the FITC image and open the image in the DAPI channel.

26. Outline a cross-section of the myocardium.

27. Click on Analyze and collect Area data.

28. Calculate the ratios of IntDen/Area (mean of fluorescence) for each heart.

29. Input all ratios in statistical analysis software.

\section{Representative Results}

Before performing the FITC-dextran assay to evaluate the changes in cardiac endothelial permeability after IR, we strongly recommend that experiments be conducted by the same researcher specializing in the establishment of myocardial IR model in rats to confirm that the coronary arteries are occluded in the same position successfully, and the ratios of ischemic area/total area are constant among all rats. As shown in Figure 1A,B, the heart (indicated by yellow arrow) was exposed, and a loop (indicated by green arrow) was formed in the myocardium located in the anterior wall of the left ventricle, approximately $2 \mathrm{~mm}$ under the edge of the left atrial appendage with a $0.5 \mathrm{~mm}$ (inner diameter) capillary silicone tube and 6-0 suture. As seen in Figure 1C, when the loop was fastened, the ischemic myocardium turned from pink to greyish pink (indicated by the white arrow) immediately.
Moreover, the myocardium reperfused and recovered its pink color immediately after the loop was loosened, as illustrated in Figure 1D. After loosening the loop, an incision was made parallel to the long axis of the femur in the inner thigh. The femoral vein was exposed and punctured with an insulin syringe, as shown in Figure 2A,B. Following a $3 \mathrm{~h}$ reperfusion, a "V" incision was made in the lower abdomen, and the aorta abdominalis and vena cava were dissected from the peritoneum (Figure 3A). Cold saline was injected into the vena cava gently, and the aorta abdominalis was cut to prevent the ventricular wall from being stretched excessively due to the intraventricular pressure that would affect its histopathological structure. Bleached lungs (red arrow in Figure 3B) and liver in Figure $3 \mathbf{C}$ indicate that the tissue was well-reperfused with saline.

Consequently, there was no significant difference in the cross-sectional area among the three groups: $1.00 \pm 0.08$ in the Sham group vs. $0.97 \pm 0.08$ in the IR group vs. $1.02 \pm$ 0.04 in the IR+TXL group (Figure 4A, $n=6$ in each group, $p>$ 0.05 between any two groups). As shown in Figure 4B,C, the mean intensity of green fluorescence in the whole sections from Sham rats was extremely low. In contrast, fluorescence emitted by extravasated FITC-dextran increased markedly in the IR group, normalized to the Sham group $(5.52 \pm 0.85$ in the IR group vs. $1.00 \pm 0.19$ in the Sham group, $n=6, p<$ 0.05). Furthermore, $T X L$ superfine powder, known to protect the cardiac microvascular endothelium ${ }^{14,16}$, remarkably reversed the higher fluorescence intensity induced by IR $(2.87 \pm 0.52$ in $\mathrm{IR}+\mathrm{TXL}$ group vs. $5.52 \pm 0.85$ in IR group, $n=6, p<0.05$ ). The images of the regional myocardium indicated that FITC-dextran overwhelmingly accumulated in the extracellular matrix but not in the microvessels, and that TXL improved endothelial barrier function (Figure 5). 
Compared with FITC-dextran, EB may be a suboptimal alternative because EB is inferior to 70,000 Da FITC-dextran in both image quality and sensitivity. Similarly, there was no significant difference in the cross-sectional area among the three groups: $1.00 \pm 0.08$ in the Sham group vs. 0.97 \pm 0.08 in the IR group vs. $0.98 \pm 0.05$ in the $I R+T X L$ group (Supplemental Figure S1A, $n=6$ in each group, $p>0.05$ between any two groups). As shown in Supplemental Figure S1B,C, the mean intensity of red fluorescence in whole crosssections from Sham rats was relatively higher, especially in the endocardium and epicardium. Although red fluorescence emitted by EB increased sharply in the IR group (4.41 \pm 0.66 in the IR group vs. $1.00 \pm 0.22$ in the Sham group, $n=6, p<$ $0.05)$, the difference between the IR+TXL and IR groups was not significant $(3.73 \pm 0.47$ in the IR+TXL group vs. $4.41 \pm$ 0.66 in the IR group, $n=6, p=0.0956$ )

Furthermore, this method was tested in a myocardial infarction (MI) model (Supplemental Figure S2). In the Myocardial Infarction Injection first (MII) group, FITC-dextran solution (at the same concentration mentioned earlier) was injected into the circulation $5 \mathrm{~min}$ before LAD ligation. This was in contrast to the FITC-dextran solution injected immediately after ligation in the MIL rats (Myocardial Infarction Ligation first) described earlier in this protocol. Interestingly, only low amounts of FITC-dextran extravasated into the ECM of the injured myocardium in $\mathrm{MI}$ rats of both groups, indicated by weak green fluorescence intensity in Supplemental Figure S2. 

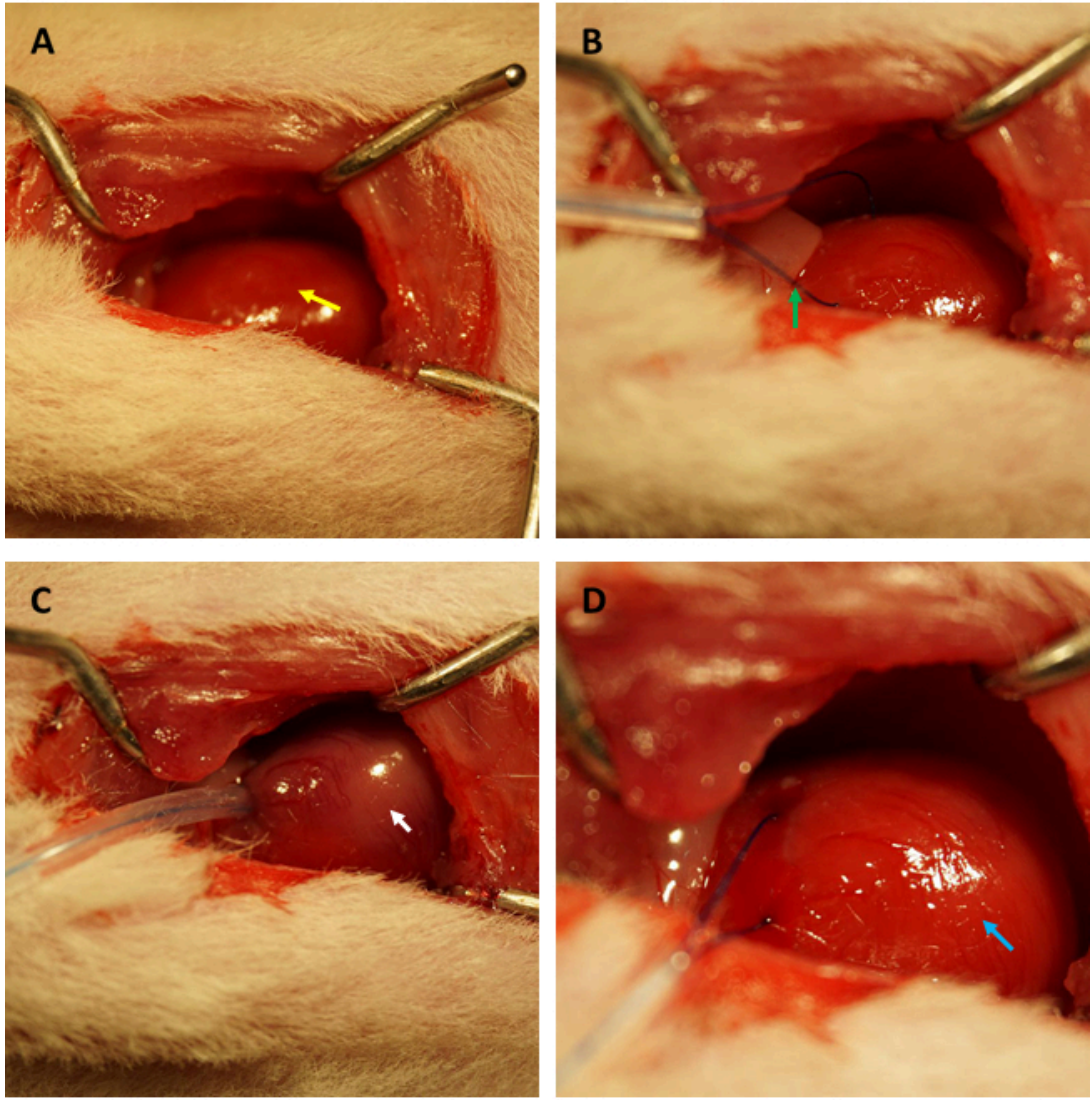

Figure 1: Visual field of thoracotomy and establishment of ischemia/reperfusion in a rat model. (A) Exposing the rat's heart (indicated by yellow arrow); (B) forming a loop to encircle the coronary artery (indicated by green arrow); (C) fastening the loop and inducing myocardial ischemia for $45 \mathrm{~min}$ (indicated by white arrow); (D) loosening the loop and allowing myocardial reperfusion for $3 \mathrm{~h}$ (indicated by the blue arrow). Please click here to view a larger version of this figure. 

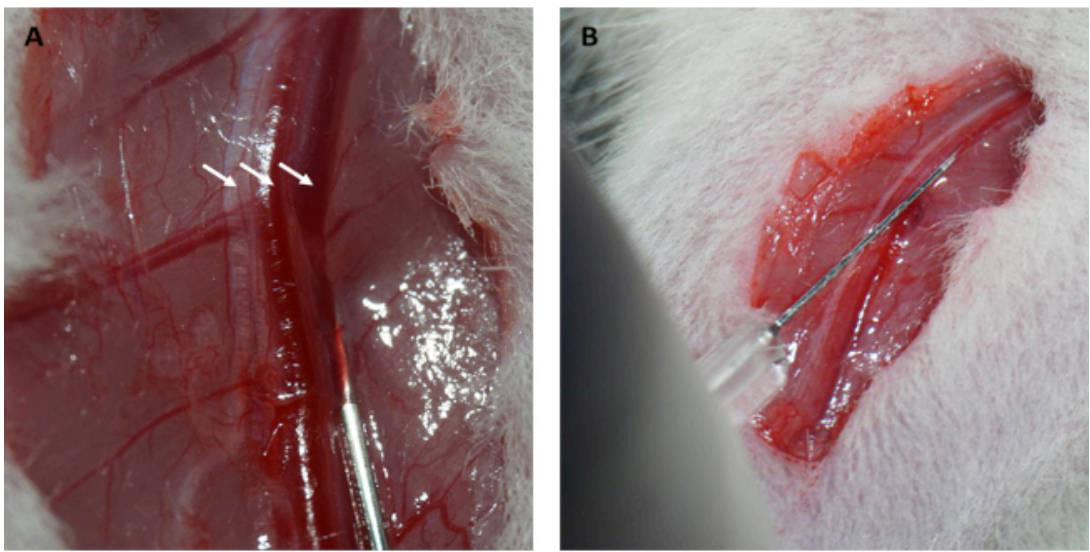

Figure 2: Dissection of the femoral vein and intravenous injection. (A) Exposing the femoral vein (the femoral nerve is indicated by the arrow on the left; the femoral artery is indicated by the arrow in the middle; the femoral vein is indicated by the arrow on the right). (B) Injecting FITC-dextran solution into the femoral vein with an insulin syringe. Abbreviation: FITC = fluorescein isothiocyanate. Please click here to view a larger version of this figure.
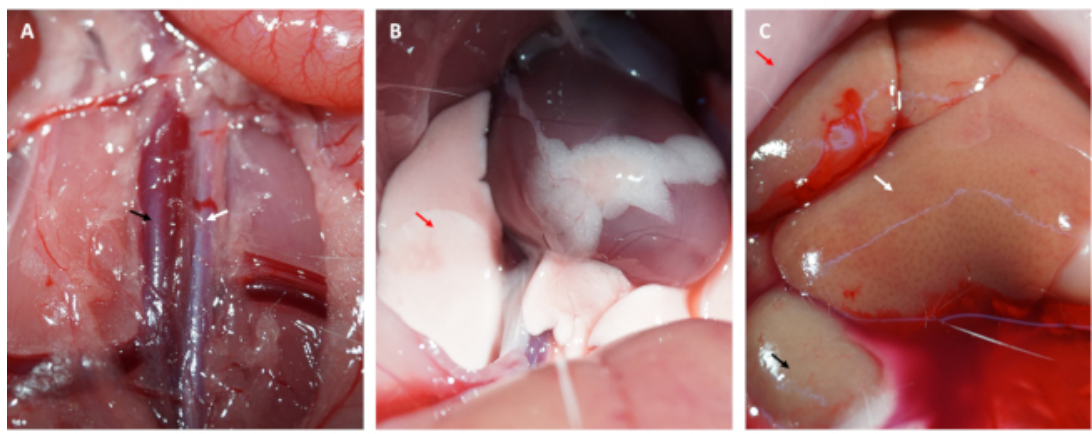

Figure 3: Tissue perfusion and symptoms of efficient perfusion. (A) Dissecting the abdominal aorta and the vena cava (the abdominal aorta is indicated by the white arrow; the vena cava is indicated by the black arrow). (B) Bleached lungs are a symptom of adequate perfusion; (C) bleached mucosa, bleached kidney, and sweating liver are all symptoms of adequate perfusion (red arrow: mucosa; white arrow: liver; black arrow: kidney). Please click here to view a larger version of this figure. 
A
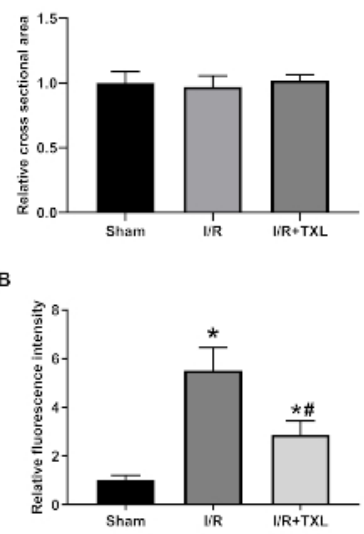
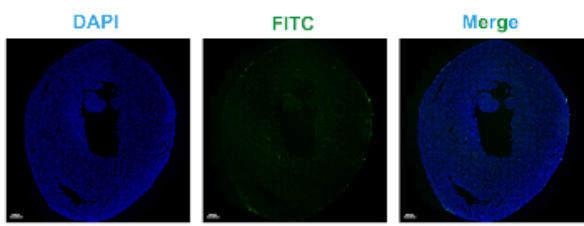

I/R
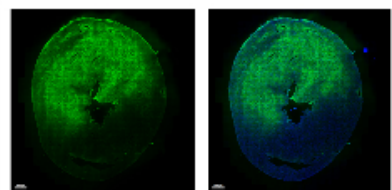

I/R+TXL
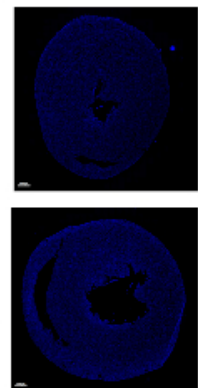
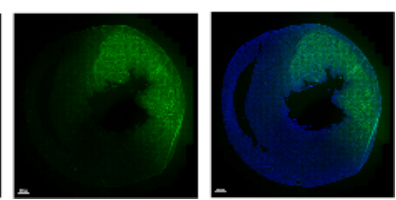

Figure 4: Representative images and analysis of mean fluorescence intensity by FITC-dextran staining. (A) Relative cross-sectional area of each group normalized to that of the Sham group ( $p>0.05)$; (B) relative fluorescence intensity in the FITC channel of each group normalized to that of the Sham group; (C) representative images at 9.5x magnification in each group. Scale bars $=800 \mu \mathrm{m}$. One-way ANOVA with Tukey's post-hoc multiple-group comparisons. * vs. Sham group, $p<0.05$; \# vs. IR group, $p<0.05$. Bar graphs show group mean $\pm S D$. Abbreviations: $I R=$ ischemia/reperfusion; $T X L=$ Tongxinluo; FITC = fluorescein isothiocyanate; DAPI = 4',6-diamidino-2-phenylindole; ANOVA = analysis of variance; SD = standard deviation. Please click here to view a larger version of this figure. 


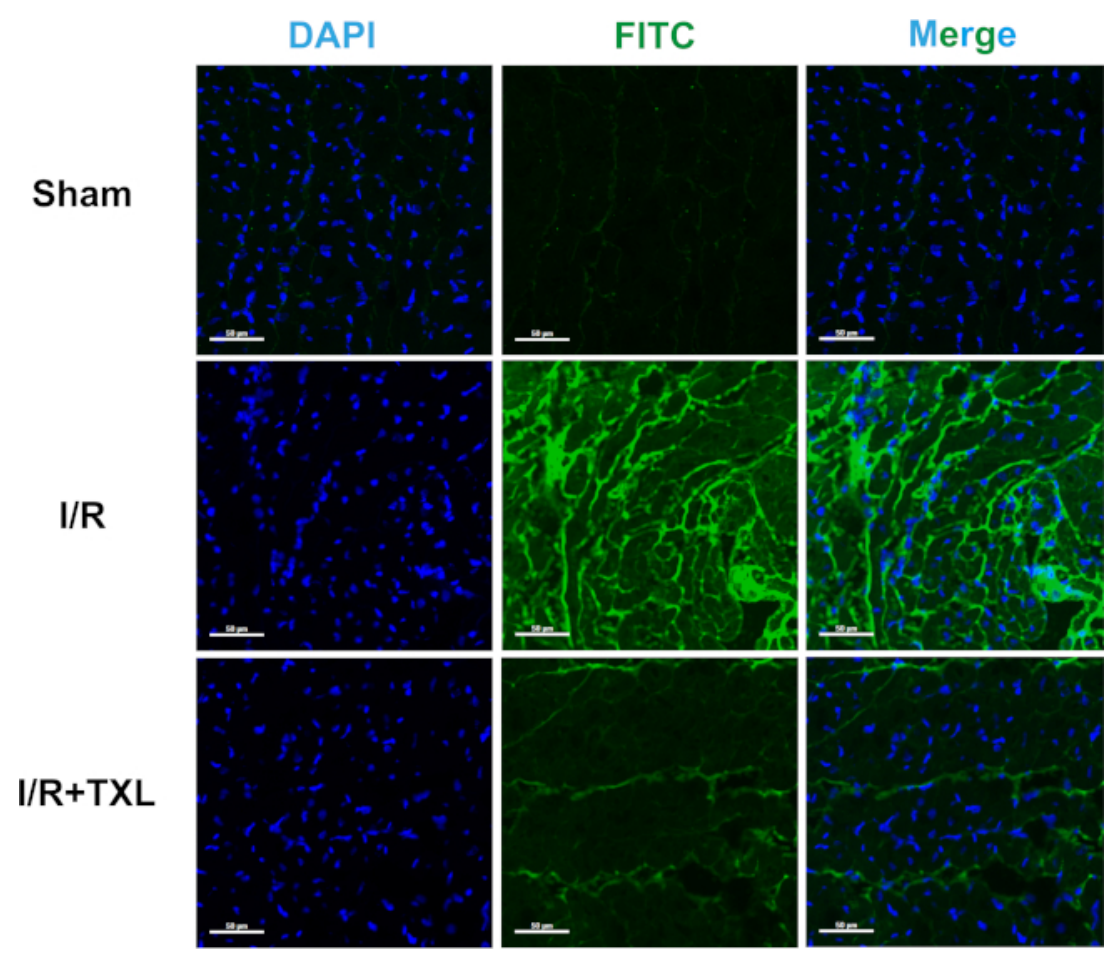

Figure 5: Representative images of areas of interest at 200x magnification of each group after FITC-dextran staining. Scale bars $=50 \mu \mathrm{m}$. Abbreviations: FITC $=$ fluorescein isothiocyanate; DAPI = 4',6-diamidino-2-phenylindole. Please click here to view a larger version of this figure.

\section{Supplemental Figure S1: Representative images and} analysis of mean fluorescence intensity by Evans Blue staining. (A) Relative cross-sectional area of each group normalized to that of the Sham group ( $p>0.05)$; $(B)$ relative fluorescence intensity in the Texas red channel of each group normalized to that of the Sham group; (C) representative images in each group. One-way ANOVA with Tukey's posthoc multiple-group comparisons. Scale bars $=800 \mu \mathrm{m}$. * vs. Sham group, $p<0.05$. Bar graphs show group mean $\pm S D$. Abbreviations: $I R=$ ischemia/reperfusion; $T X L=$ Tongxinluo; DAPI $=$ 4',6-diamidino-2-phenylindole; ANOVA = analysis of variance; SD = standard deviation. Please click here to download this File.
Supplemental Figure S2: Representative images at 9.5x magnification of FITC-dextran staining in a myocardial infarction model. Scale bars $=800 \mu \mathrm{m}$. Abbreviations: $\mathrm{MII}=$ myocardial infarction injection first (inject FITC-dextran before myocardial infarction); MIL = myocardial infarction ligation first (inject FITC-dextran immediately after myocardial infarction); FITC = fluorescein isothiocyanate; DAPI = 4',6-diamidino-2phenylindole. Please click here to download this File.

\section{Discussion}

This paper presents a protocol to assess the dysfunction of the cardiac endothelial barrier after being exposed to MIRI. It should be noted that there are several crucial steps in this protocol that determine the reliability and accuracy of the 
results. All steps involving FITC-dextran are to be performed in a dark room. The FITC-dextran/saline solutions must be stored at $4{ }^{\circ} \mathrm{C}$ and wrapped with tin foil. Additionally, coronary arteries must be ligated, as illustrated in Figure 1, so that the myocardium can be reperfused immediately after the loop is loosened. Researchers can choose $5-0$ or $6-0$ polyethylene sutures because thinner strings under $7-0$ are more likely to cut myocardial fibers and vessels. Importantly, the tracer solution should be injected into the femoral veins and not the tail veins. Each heart should be well-perfused and wellrinsed with ice-cold saline before and after being sacrificed, respectively.

Many researchers specializing in MIRI are inclined to study the disrupted subcellular structure of the endothelium in the earlier stage of myocardial reperfusion-the formation of apoptotic bodies, swelling mitochondria, and abnormal intercellular junctions-to prove the hyperpermeability of the endothelium via electron microscopic examination. Immunoblotting has also been used to measure microvascular permeability because the decrease in levels of tight conjunction proteins is regarded as the core mechanism of endothelial injury and malfunction of endothelial barrier over time. For instance, VE-cadherin ${ }^{20}$ and $\beta$-catenin ${ }^{21}$ are key elements of endothelial cell-to-cell adhesion junctions, and these connections contribute to the maintenance of vascular integrity ${ }^{22}$. Tight junctions, such as JAM- $\mathrm{A}^{23,24}$ and occludin $^{25}$, also cooperatively participate in the construction of the endothelial barrier in the circulatory system.

Currently, an increasing number of studies suggest that mitochondrial function and mitochondrial homeostasis could be key targets of protective pathways against reperfusion injury. As reported in pathophysiological and pharmacological studies, mitophagy can play an important role in the cellular death of the endothelium and myocardium induced by $\mathrm{IR}^{26}$. In addition, mitochondrial fission, mitochondrial proteostasis, and mitochondrial quality control may protect high-risk myocardium in reperfused areas ${ }^{27,28}$. The above methods focus more on demonstrating different mechanisms or causes of hyperpermeability than on displaying endothelial permeability directly and visually. Moreover, sample preparation for electron microscopic examination is complicated and dangerous. Glutaraldehyde, acetone, and osmic acid solutions used in electron microscopy probably volatilize and erode the mucosa, conjunctiva, and skin, even threatening lives if used in uncontrolled environments.

Compared to these indirect and life-threatening methods, this method, which measures the fluorescence intensity emitted by FITC-dextran extravasation, is ideal for assessing cardiac microvascular permeability. First, 70,000 Da FITCdextran cannot penetrate through the endothelium under normal physiological conditions but can enter the myocardial interstitial space under IR, which allows a clear differentiation between rats under different treatments. Additionally, the FITC-dextran solution is injected into the blood via the femoral veins, which guarantees that all of the fluid is injected into the rat's circulation. In contrast, tail vein injection is more difficult in rats because the tail vein is indistinct and covered with a thick stratum. Further, unlike regular paraformaldehyde fixation and paraffin embedding, myocardial tissue treatment is simplified and optimized using the quick-freeze pathological section technique used in this study. Short storage time, light-proof environment, and cryopreservation may together alleviate fluorescence quenching of FITC.

More importantly, this method can minimize selection bias during statistical analysis. We perform sufficient heart perfusion with saline to ensure that nearly all the blood mixed 
with FITC-dextran is douched out from the myocardium and the heart chambers, which can eliminate the fluorescence emitted by any FITC remaining in the vessels. If this is not the case, fluorescence merged with capillaries should be excluded and trimmed artificially. While collecting tissue from areas of interest, no visual boundary was observed between the ischemic myocardium and the remote myocardium, especially after EB dye staining. This hindered the collection of samples from these representative areas. Using the method described herein, researchers can obtain images of whole sections and analyze the fluorescence intensity using a quantitative slider scanner.

Interestingly, EB dye can be an alternative, albeit suboptimal, to FITC-dextran. Compared with the relatively harsh storage conditions of FITC-dextran, EB powder or its solution can remain stable at room temperature for a long time. Additionally, EB is relatively inexpensive for most institutions. Nonetheless, certain disadvantages could limit its application. First, EB staining shows lower sensitivity than FITC-dextran, which may underestimate the differences among groups and increase the sample size of each group. Besides, EB shows stronger adhesion to proteins. The endocardium and epicardium are more easily stained by EB, which can influence the results of fluorescence density. Further, obvious filling defects in the reperfusion area remarkedly reduce the image quality, which can limit the use of EB.

To extend the application of this method in an MI model, the method was tested in an MI model. First, FITC-dextran was injected into blood vessels after LAD ligation. As expected, a low level of fluorescence was emitted from the slice because FITC-dextran in blood flow cannot pass through the ligated site, and no FITC-dextran entered the ischemic area. FITCdextran was also applied 5 min before ligation to ensure the passage of FITC-dextran into all organs. Consequently, FITCdextran failed to penetrate the microvascular endothelium probably because no perfusion pressure in ligated blood vessels can drive FITC-dextran through the endothelium. Generally, this method probably requires significantly more modification to satisfy its application in the MI model.

Despite the above advantages, there are limitations to this method. First, this protocol was not tested in IR models of other animals, which may limit its application in preclinical and clinical studies. Second, FITC-labeled dyes are more expensive than other common dyes such as EB. However, unlike EB, as $10 \%$ w/v FITC-dextran solution, diluted with serum, rarely changes the color of the myocardium, FITCdextran staining and EB/2,3,5-triphenyltetrazole chloride (TTC) dual staining may be conducted in the same heart, which can trim the budget with respect to the number of animals and the dyes used. Last, the slide scanner used in this study-the Quantitative Slide Scanner and similar instruments-may not be available in some laboratories. In summary, this protocol presents a simplified, reliable, and visual method for the detection of cardiac microvascular endothelial permeability in an IR model in rats using a fluorescence assay based on an automated quantitative pathology imaging system. This protocol demonstrates that 70,000 Da FITC-dextran performs better than EB in the functional assessment of the endothelial barrier of cardiac microvessels after IR injury.

\section{Disclosures}

The authors declare no conflicts of interest.

\section{Acknowledgments}

This study was supported by grants from The National Key Research and Development Program of China (No. 
2017YFC1700503) and the National Natural Science Foundation of China (No. 81874461 and No. 81774292).

\section{References}

1. Yellon, D. M., Hausenloy, D. J. Myocardial reperfusion injury.New England Journal of Medicine. 357 (11), 1121-1135 (2007).

2. Hausenloy, D. J. et al. The coronary circulation in acute myocardial ischaemia/reperfusion injury: a target for cardioprotection.Cardiovascular Research. 115 (7), 1143-1155 (2019).

3. Tada, Y., Yang, P. C. Myocardial edema on T2-weighted MRI: new marker of ischemia reperfusion injury and adverse myocardial remodeling.Circulation Research. 121 (4), 326-328 (2017).

4. Chandrasekar, B., Smith, J. B., Freeman, G. L. Ischemiareperfusion of rat myocardium activates nuclear factor-KappaB and induces neutrophil infiltration via lipopolysaccharide-induced CXC chemokine. Circulation. 103 (18), 2296-2302 (2001).

5. Li, X. -D. et al. Tongxinluo reduces myocardial noreflow and ischemia-reperfusion injury by stimulating the phosphorylation of eNOS via the PKA pathway.American Journal of Physiology. Heart and Circulatory Physiology. 299 (4), H1255-61 (2010).

6. Pei, $H$. et al. TNF- $\alpha$ inhibitor protects against myocardial ischemia/reperfusion injury via Notch1mediated suppression of oxidative/nitrative stress. Free Radical Biology \& Medicine. 82, 114-121 (2015).

7. Liao, $\mathrm{Y}$. $-\mathrm{H}$. et al. Interleukin-17A contributes to myocardial ischemia/reperfusion injury by regulating cardiomyocyte apoptosis and neutrophil infiltration. Journal of the American College of Cardiology. 59 (4), 420-429 (2012).

8. Pedersen, S. F. et al. Assessment of intramyocardial hemorrhage by T1-weighted cardiovascular magnetic resonance in reperfused acute myocardial infarction.Journal of Cardiovascular Magnetic Resonance. 14 (1), 59 (2012).

9. Fang, L. et al. Differences in inflammation, MMP activation and collagen damage account for gender difference in murine cardiac rupture following myocardial infarction.Journal of Molecular and Cellular Cardiology. 43 (5), 535-544 (2007).

10. Xu, Y. et al. Quantifying blood-brain-barrier leakage using a combination of evans blue and high molecular weight FITC-Dextran.Journal of Neuroscience Methods. 325, 108349 (2019).

11. $\mathrm{Li}, \mathrm{B}$. - R. et al. In vitro and in vivo approaches to determine intestinal epithelial cell permeability.Journal of Visualized Experiments: JoVE. 140, 57032 (2018).

12. Yao, L., Xue, X., Yu, P., Ni, Y., Chen, F. Evans Blue dye: a revisit of its applications in biomedicine. Contrast Media \& Molecular Imaging. 2018, 7628037 (2018).

13. Roberts, L. N. Evans blue toxicity. Canadian Medical Association Journal. 71 (5), 489-491 (1954).

14. Qi, K. et al. Tongxinluo attenuates reperfusion injury in diabetic hearts by angiopoietin-like 4-mediated protection of endothelial barrier integrity via PPAR- $\alpha$ pathway.PLoS One. 13 (6), e0198403 (2018).

15. Zhang, H. -T. et al. No-reflow protection and long-term efficacy for acute myocardial infarction with Tongxinluo: a randomized double-blind placebo-controlled multicenter 
clinical trial (ENLEAT Trial).Chinese Medical Journal (English). 123 (20), 2858-2864 (2010).

16. Cui, $H$. et al. Induction of autophagy by Tongxinluo through the MEK/ERK pathway protects human cardiac microvascular endothelial cells from hypoxia/reoxygenation injury.Journal of Cardiovascular Pharmacology. 64 (2), 180-190 (2014).

17. Chen, G. $-H$. et al. Inhibition of miR-128-3p by Tongxinluo protects human cardiomyocytes from ischemia/reperfusion injury via upregulation of $p 70 \mathrm{~s} 6 \mathrm{k} 1 /$ p-p70s6k1.Frontiers in Pharmacology. 8, 775 (2017).

18. $\mathrm{Yu}, \mathrm{Z}$. $-\mathrm{H}$. et al. PI3K/Akt pathway contributes to neuroprotective effect of Tongxinluo against focal cerebral ischemia and reperfusion injury in rats. Journal of Ethnopharmacology. 181, 8-19 (2016).

19. Yang, H. -X., Wang, P., Wang, N. -N., Li, S. -D., Yang, M. -H. Tongxinluo ameliorates myocardial ischemiareperfusion injury mainly via activating Parkin-mediated mitophagy and downregulating ubiquitin-proteasome system. Chinese Journal of Integrative Medicine. (2019).

20. Pulous, F. E., Grimsley-Myers, C. M., Kansal, S., Kowalczyk, A. P., Petrich, B. G. Talin-dependent integrin activation regulates VE-cadherin localization and endothelial cell barrier function. Circulation Research. 124 (6), 891-903 (2019).

21. Cong, X., Kong, W. Endothelial tight junctions and their regulatory signaling pathways in vascular homeostasis and disease.Cellular Signaling. 66, 109485 (2020).

22. Giannotta, M., Trani, M., Dejana, E. VE-cadherin and endothelial adherens junctions: active guardians of vascular integrity.Developmental Cell. 26 (5), 441-454 (2013).
23. Monteiro, A. C., Parkos, C. A. Intracellular mediators of JAM-A-dependent epithelial barrier function.Annals of the New York Academy of Sciences. 1257, 115-124 (2012).

24. Kakogiannos, N. et al. JAM-A acts via C/EBP$\alpha$ to promote Claudin-5 expression and enhance endothelial barrier function.Circulation Research. 127 (8), 1056-1073 (2020).

25. Feldman, G. J., Mullin, J. M., Ryan, M. P. Occludin: structure, function and regulation.Advanced Drug Delivery Reviews. 57 (6), 883-917 (2005).

26. Zhou, $H$. et al. NR4A1 aggravates the cardiac microvascular ischemia reperfusion injury through suppressing FUNDC1-mediated mitophagy and promoting Mff-required mitochondrial fission by CK2a.Basic Research in Cardiology. 113 (4), 23 (2018).

27. Wang, J., Toan, S., Zhou, H. Mitochondrial quality control in cardiac microvascular ischemia-reperfusion injury: New insights into the mechanisms and therapeutic potentials.Pharmacological Research. 156, 104771 (2020).

28. Zhu, H. et al. Phosphoglycerate mutase 5 exacerbates cardiac ischemia-reperfusion injury through disrupting mitochondrial quality control.Redox Biology. 38, 101777 (2021). 